\title{
The Innovation and Construction of Enterprise Information Management Mode in the Era of Cloud Computing
}

\author{
Lijiao \\ Xin Xiang University, Xin Xiang, China, 453000, China \\ jonahlee369@163.com
}

Keywords: cloud computing, information management mode, innovation

\begin{abstract}
The main purpose of writing this paper is to introduce the application of a new technology and business model--cloud computing to enterprises which are undertaking or will undertake the construction and operation of information management system, in order to solve the problems of high investment and low efficiency in information system construction and operation. The paper makes a general introduction to cloud computing, discusses the influence of cloud computing on enterprises taking into account the actual problems of enterprises, and puts an emphasis on how to use cloud computing for information management innovation. The paper puts forward a brand-new mode of enterprise information management - cloud services and points out the commercial application value of cloud computing, and enterprises can use the ideas in this paper for important reference in the process of information management innovation.
\end{abstract}

\section{Thoughts and ideas of cloud computing}

From the technical point of view: Cloud computing is a kind of distributed computing technology, it automatically divides the huge computational processing program into numerous smaller subroutines through the network, and then handed them over to the huge system composed of multiple servers, and the processing results are sent back to the user after search, compute and analysis. By means of this technology, a network service provider can process millions or even billions of information in several seconds, and achieve equally strong performance in network service as a "supercomputer".

From the application point of view: cloud computing is a commercial evolution outcome of "Grid Computing", and it is a new business model of IT. By signing a contract with the services provided by the cloud, a user can complete the compute task with the hardware or software provided by the cloud with minimum investment, thus realize commercial value for users. The user mentioned herein not only refers to the individual, but also refers to enterprise or company, of course, more often refers to the latter. Cloud computing refers to commercialized service realization mode of information management provided by the cloud for the enterprise or company.

Cloud computing has the following characteristics:

1. Large-scale, cloud computing often has hundreds of thousands or even millions of servers to make up the cloud, and the computing center is composed of ultra-large-scale CPU arrays;

2. Virtualization, cloud computing supports the user to get the services in any location and with a variety of terminals. The requested resources come from the cloud rather than fixed tangible entities;

3. Reliability, the cloud uses fault-tolerance in multiple copies of data and interchange ability of computational node with the same structure to ensure reliability of service. Using cloud computing is more reliable than using a local computer;

4. Universality. Cloud computing is not tailored for specific applications, we can construct various applications with the support of the cloud, and the same cloud can support different applications;

5. Extensibility, the scale of the cloud can change dynamically to meet the application need and the growth of user scale;

6. On-demand service, the cloud is a vast pool of resources. Users can buy services according to their own need; 
7. Cheapness, special fault-tolerant measures make it possible to use extremely inexpensive nodes to form clouds, automated management makes the management of data center cost much lower. the universality makes the utilization rate of resources rise considerably. Therefore, the cloud has very high cost performance.

Obviously, through the above description of cloud computing, our understanding cannot just focus on the shallow level of the update of equipment and technology. For cloud computing, the object that can better reflect its value is not itself but the new commercial application mode built on the basis of it, namely the "cloud service". The changes that the cloud brings to business and other aspects determine the economic attributes of the cloud. At this level, the cloud services are far more competitive than traditional services. It is the economic attribute of cloud computing to determine that the cloud computing becomes a new productive force. It can be thought that cloud computing is the concentrated embodiment of the innovation of information technology application mode and service mode. Among them, innovation is the characteristic of cloud computing and service is the soul of cloud computing. The application of cloud computing will bring profound even revolutionary changes to the world. A modern enterprise has to bear the brunt of the changes and go on the road of information management reform.

\section{Cloud computing reforms information management}

There are mainly two types of strategies of enterprise information system construction:

The first strategy is to establish system by enterprises. First of all, enterprises set up informatization construction team and analyze business process; then, they formulate the plan and carry it out, specific works include procurement, deployment, installation, debugging and training of IT hardware and software; finally, they operate and manage the system. The main disadvantages of this strategy are large investment, low efficiency and long cycle. First, the continuously investment of capital lead to a rapid increase in business costs; Second, the resources cannot be effectively integrated, the utilization rate of resources keeps on dropping, application request within the system cannot be reasonably distributed by resources, all these things result in substantial waste of resources; Third, the poor communication condition and even incompatibility in the process of system management result in repetitive work, which greatly reduce the efficiency. With the continued expansion of enterprise size, the increasing business processes and the increasing number of customers, the original information management system will surely not fully meet the requirements of development. If enterprises expand blindly and disorderly without fully rational planning, the complexity and the error rate will surely further increase in managing the system. Thus, the more expansion, the more chaos, it is an endless vicious circle.

The second strategy is outsourcing, that is, the software and hardware procurement, management and operation of information system are outsourced to a third-party professional IT institution, thus enterprises separate themselves from the management work. Compared with the first strategy, system outsourcing greatly reduces the investment, and makes enterprises focus on core business. However, there are also disadvantages by doing so, the extension of system is subject to IT institutions, enterprises cannot establish the system completely in accordance with their own wishes, and the IT system of the third party will not fully suit the characteristics and need of enterprises.

So, can we find an eclectic method? The answer is the innovative application of cloud computing. By doing so, information system can completely fit the demand of the enterprise without too many investments. In the mode of cloud computing, enterprises can obtain infrastructure services (IaaS), platform services (PaaS) and software services (SaaS) at the same time. The enterprise can access, compute and store network resources anytime and anywhere through the Internet, and it can intelligently distribute storage and computation resources according to service demand. So, the operation of enterprise data center in the mode of cloud will be more similar to the Internet. The resources and services are all placed in the cloud with a high degree of concentration, thus forming application modules that can be arbitrarily split and combined. This enables the enterprise to switch resources to any desired application flexibly, the enterprise accesses 
the data and gets services according to demand, thus the win-win of efficiency and cost is achieved. The specific effects that cloud computing bring to enterprise information management are as follows:

First of all, cloud computing can greatly improve the efficiency of enterprise management and service level. The enterprise using cloud computing does not need to develop corresponding software or to provide corresponding service platform. it only needs to pay a small rent for realizing the modernization of information management.

Secondly, cloud computing can reduce the cost of enterprise IT operation and maintenance to the maximum extent. In cloud computing, the enterprise only needs to customize corresponding services, the cloud service providers or the cloud provide basic structure, services and software and hardware resources. This practice can save cost. there is no need to purchase expensive hardware and to shoulder frequent maintenance and upgrade. The enterprise does not need to establish their own data centers, large computer room and server center. the maintenance and operation costs of hardware and software are low.

Thirdly, cloud computing can greatly improve utilization of enterprise resources. Enterprises can set up their cloud computing infrastructure by themselves, and they can also complete the computation, storage and platform services by the way of renting the cloud when there is a need. Several enterprises sharing the cloud computing infrastructure not only saves cost while improving the resource utilization rate, but also saves resources in meeting the gradually increasing demand.

Fourthly, cloud computing enlarges the extension of enterprise hardware and software application and changes the application mode of hardware and software products. With cloud computing, business users do not need to purchase new servers and to deploy software, they can get application environment or application itself with customization and renting. One of the pioneers of cloud computing - Google even emphasized, almost all software can be placed on the Internet and service will replace software in the future.

Finally, for the long-term strategic development of enterprises, the IT departments in the enterprises in the era of the cloud no longer need to worry about the constant upgrade of servers and software and other computational problems. It is just like renting a house, the enterprise is only in charge of using, the cloud will do all the other things. Thus, IT departments are completely emancipated, and they can do more about innovation. Enterprises can obtain external information more easily and can use any languages to communicate with customers and partners so as to help enterprises make decisions.

\section{Constructing new management system architecture taking cloud computing as the core}

If the enterprise wants to build cloud computing architecture, it must consider three questions, they are the SOA, the three-layer services of the cloud, the choice between private cloud and public cloud.

1. Introduction of the concept SOA

The concept of SOA appeared earlier than the concept of cloud computing. SOA stands for service-oriented architecture. it is a method of building a distributed system application. It sends the functions of applications to the end-user or other services as a service. SOA uses open standards, interacts with software resources and adopts the standard way of indication. SOA is the evolution and replacement of object-oriented architecture model.

The core idea of SOA is that each business system of an enterprise is no longer independent, the systems can easily contact each other and share information, reuse the previous information software. Its ultimate goal is to increase business agility through building integration capacity in distributed, large-scale and heterogeneous environment. The SOA can solve the problem of application integration of different IT systems within an enterprise in a heterogeneous environment, that is, collaborative software based on SOA is able to integrate IT and operations of the enterprise such as data from heterogeneous systems like ERP, CRM and HR. This idea coincide with that of cloud computing. 
SOA should learn the following ideas from cloud computing:

(1). Design of Service Architecture

Because of having been carefully designed, various business services related to cloud computing are very distinctive and attractive, such as the Amazon Elastic Compute Cloud and Simple Storage Service, as well as the elastic compute, cloud engine and data storage and compute services provided by Aliyun.com. Moreover, these cloud services are indeed able to meet your needs. In contrast, many SOA projects are too rough and complicated. So, the SOA designing of enterprises can draw lessons from the thought of cloud services.

(2). Flexible services

One of the most important features of cloud computing services is the ability to stretch anytime when need, e.g., the elastic compute of cloud services. In the cloud clusters, computing unit uses virtualization technology to make the whole cluster work together. For a certain application, the actual occupied computing resources in a cluster are elastic. multiple physical backups are made to save the data in real time. The amounts of system computation required by different-sized enterprises are different. the amounts of system computation required by one enterprise in different departments and circumstances are also different. Enterprises can adjust computational resources according to their need at any time to improve service extensions. However, extending fixed services is usually difficult in SOA. the flexibility of the service is worth paying attention to when building SOA.

A good SOA will lead to a good cloud computing strategy, and then reduce the cost, enhance the flexibility of enterprise computing. The integration point of cloud computing and SOA is that the enterprise can consider cloud computing to be an extension of SOA delivering resources to cloud, such as storage as a service, data as a service, platform as a service. The key is to determine what services, information and processes are the best objects to be put in cloud. Therefore, enterprises can take cloud as an additional platform for running applications and services. Thus, the enterprise does not need to place a server full of software in a data center and to hire some professionals for its maintenance.

2. The three-layer service model of the cloud

The complete cloud computing service architecture is composed of three layers of cloud service models. They are cloud software (SaaS), cloud platform (PaaS) and cloud equipment (IaaS) from top to bottom.

The layer of cloud software refers to software services. It is a mode of providing software through the network. It does not require users to purchase the software, but users need to rent software based on web from provider in order to manage business activities. The layer of cloud platform refers to platform service. The platform service is providing software development platform in the mode of SaaS. The layer of cloud equipment refers to infrastructure services, it will Integrate basic equipment (such as an IT system, database, etc.), and the service is obtained through the Internet.

Establishing cloud architecture by enterprises is to integrate IT resources of different layers and to form a service pattern, then offer the service to employees, customers and suppliers for their use. Finally, a mode of innovation is formed.

First of all, the IT infrastructure forms a sharing of resources, using the virtual technology, massive storage calculation, load balancing and dynamic resource pools, realizing IT resources being concentrated to the upper level to the maximum extent;

Secondly, improving data integration, unifying data standards and models, modularizing data processing procedures;

Third, realizing data integration, process integration and application integration based on SOA;

Fourth, based on the BI, analytical ability, presentation skills and integrated portal, put all the data and information resources together, show them to the employees, customers and suppliers.

Finally, based on the demand of services, gradually establish an enterprise cloud, forming a dynamic pattern, a virtual cluster and an open software structure, realizing simplified management and unified control optimization, reducing the risk. 
However, at present, not all the cloud service providers in commercial area can provide a complete solution, especially for smaller users and markets. Therefore, it is not to say that all the current enterprises must choose all the three services. Different enterprises have different choices. For example, the small and medium-sized enterprises are more likely to choose SaaS and part of PaaS services, large enterprises are more likely to choose all.

3. Private cloud and public cloud

In view of the information security, an enterprise usually divides its information system into two parts and separates them by a firewall. Therefore, there are private cloud and public cloud when building cloud architecture.

The private cloud is built inside the firewall of the enterprise. The cloud technology assists enterprise information management, serves each department and business within the enterprise. The public cloud is set up outside the firewall of the enterprise. It is a product of social division of labor and specialization,i.e. the IT service architecture built by professional third party cloud service providers for enterprises. In the public cloud, each enterprise is a component and a beneficiary of the cloud.

Choosing to build private cloud or public cloud by an enterprise is related to the industry that the enterprise is engaged in, enterprise size, application types and other factors.

(1). Infrastructure selection.

When enterprises construct private cloud platform, IT infrastructure is their own. It is located in the enterprise. While adopting public cloud platform, IT infrastructure is located in a third-party data center.

(2). Infrastructure integration

In earlier years, the construction and technological evolution of enterprise IT often feature different technologies and platforms. That is to say, the platform environment is heterogeneous, the compatibility is bad. However, the platform of public cloud service provider is built on low-cost and standard hardware platform. It has good cost performance, but also meets most needs of users. In addition, in providing services, the service and environment of public cloud service providers are often isomorphic, and it is different from the special IT conditions which enterprises set up by themselves.

(3). Construction and usage costs.

Constructing enterprise private cloud requires one-time large investment to purchase software and hardware equipment, including data center infrastructure. It is a large fixed cost. If the enterprises adopt public cloud services, enterprises will pay for it according to the amount of service resource usage, the enterprise does not need to invest heavily at an early stage to use IT services.

(4). The management authority.

The enterprise has its own private cloud built by itself, and has its own team to control it completely. enterprise can carry out a transformation according to the actual need. The benefits of public cloud services are that the enterprise does not need to manage basic platform services, but the ability of enterprise customization is also reduced.

Large enterprises are sensitive to security. At the same time, large enterprises generally have had large investment in the IT construction. Therefore, in order to protect investment, enterprises will continue to maintain the current computing environment, but they should reform the existing environment according to cloud computing theory and technology. Therefore, large enterprises like to choose private cloud.

The small and medium enterprises are sensitive to cost, and they are unlikely to have the necessary skills to build a complete cloud computing platform, so they will think more about public cloud services. From a certain point of view, we can say that the public cloud gives small and medium enterprises an opportunity of standing in the same competition level with large enterprises.

Private cloud is closely related with public cloud. In the early stage of cloud platform construction, the private cloud is the foundation of public cloud, with the development of application, private and public cloud resources will be mixed and used alternately; subsequently, the 
proportion of public cloud services will be more and bigger; finally, the public cloud will play an important role in enterprise IT service. Among them, in a long period of time, the enterprise will use a mixed cloud computing services.

At present, the original enterprise information management experience has offered lots of accumulation and innovation for the developing of private cloud. For most enterprises, private cloud is the feasible implementation plan in the future five years; With regard to establishing public clouds, some world-famous IT and Internet companies have put forward their own solutions respectively, but for the whole society at present, public cloud is not very mature. From accepting, getting familiar with to implementing, it is a long-term process, there is no mature concept that can guide, and it is a social work that cannot be done by a single company.

\section{References}

[1] Zhixiong Yang, Cloud computing makes enterprise future full of imagination, J. Today UF, (2011.2) 82-83.

[2] Leiyun Wan, From the traditional IT to cloud strategy, J. Today UF, (2011.4) 48.

[3] Gruman, Galen, What cloud computing really means, J/OL. http://www.infoworld.com/d/cloud-computing/what-cloud-computing-really-means-031, (2008, 4).

[4] Baidu Library, Google cloud computing principle, DB/OL. http://wenku.baidu.com/view/149451b069dc5022aaea006b.html, 2-5. 